Final publication is available from Wolters Kluwer Health/Lippincott Williams \& Wilkins publishers https://doi.org/10.1097/HTR.0000000000000016

\title{
Absence of differences between male and female adolescents with prior sport concussion
}

\author{
Brian L. Brooks, PhD* \\ Departments of Paediatrics and Clinical Neurosciences, Faculty of Medicine, University of Calgary \\ Alberta Children's Hospital Research Institute for Child \& Maternal Health, University of Calgary \\ Calgary, Alberta, Canada \\ Martin Mrazik, PhD \\ Department of Educational Psychology, University of Alberta \\ Edmonton, Alberta, Canada \\ Karen M. Barlow, MB, ChB, MRCPCH \\ Paediatric Neurology, Faculty of Medicine, University of Calgary \\ Neurosciences (Brain Injury and Rehabilitation programs), Alberta Children's Hospital \\ Alberta Children's Hospital Research Institute for Child \& Maternal Health, University of Calgary \\ Calgary, Alberta, Canada \\ Carly McKay, PhD \\ Sport Injury Prevention Research Centre, Faculty of Kinesiology, University of Calgary \\ Calgary, Alberta, Canada \\ Willem H. Meeuwisse, MD, PhD \\ Sport Injury Prevention Research Centre, Faculty of Kinesiology, University of Calgary \\ Calgary, Alberta, Canada \\ Carolyn A. Emery, PhD \\ Sport Injury Prevention Research Centre, Faculty of Kinesiology, University of Calgary \\ Alberta Children's Hospital Research Institute for Child \& Maternal Health, University of Calgary \\ Calgary, Alberta, Canada \\ *Correspondence: \\ Brian L. Brooks, Ph.D. \\ Neurosciences program, Alberta Children's Hospital \\ 2888 Shaganappi Trail NW, Calgary, Alberta, Canada T3B 6A8 \\ Phone: 1-403-955-2597 \\ Fax: 1-403-955-7501 \\ Electronic email: brian.brooks@albertahealthservices.ca
}

Manuscript word count: $3192(\max 4500)$

Author Disclosure Statements

None of the authors have a financial interest in the ImPACT battery. BLB receives funding from a test publisher (Psychological Assessment Resources, Inc.), book royalties from Oxford University Press, 
and in-kind test credits for research from another computerized test publisher (CNS Vital Signs). In-kind support from ImPACT was provided to MM for the assessments conducted in Edmonton.

\section{Funding Acknowledgements}

Computerized cognitive testing in this study was funded in Calgary by the McCarthy Tetrault award (administered by the Alberta Children's Hospital Research Institute for Child \& Maternal Health on behalf of the Alberta Children's Hospital Foundation) to BLB for a computerized neurocognitive laboratory (computers, test credits) and in Edmonton by funding provided to $\mathrm{MM}$ and in-kind support by ImPACT to MM. The Sport Injury Prevention Research Centre is one of the International Research Centres for Prevention of Injury and Protection of Athlete Health supported by the International Olympic Committee. WHM and CAE acknowledge funding from Alberta Innovates Health Solutions (AIHS), Canadian Institutes of Health Research (CIHR), and the Alberta Children's Hospital Research Institute for Child \& Maternal Health (ACHRI; Professorship in pediatric rehabilitation for CAE). None of the funding sources were involved in design and conduct of the study; collection, management, analysis, and interpretation of the data; or preparation, review, or approval of the manuscript.

\section{Acknowledgements}

The authors thank the following people for their help with data collection and data management (alphabetical order): Tracy Blake MSc, Andrea Jubinville MA, Nicole Lemke, Maria Romiti, and Kathryn Schneider PhD. The authors thank Helen Carlson PhD for her help with entering the demographicallyadjusted normative scores and formatting the manuscript. Thanks to Hockey Calgary, Females Hockey Calgary, Hockey Edmonton, Edmonton Females Hockey, the Edge School (Calgary), the team designates, research assistants, coaches, families, and the players who participated in this study. 


\begin{abstract}
Objective: Sex differences following concussion are poorly understood. The purpose of this study was to examine whether male and female adolescent athletes with prior concussions differ regarding neurocognitive function and symptom reporting.

Setting: Community-based hockey teams.

Participants: Participants included 615 elite hockey players $13-17$ years old (mean=15.5, 95\% Cl=15.415.6). There were 517 males and 98 females. Players with English as a second language, attention or learning problems, a concussion within six months of baseline, or suspected invalid test profiles were excluded from these analyses.

Design: Cross-sectional.

Main Measure: Domain scores and symptom ratings from the ImPACT computerized battery. Results: There were no significant neurocognitive differences between males and females with $[F(5,227)=1.40, p=0.227]$ or without $[F(5,376)=1.33, p=0.252]$ a prior history of concussion. Male and female athletes with a history of concussion reported higher raw symptom scores than those without a prior concussion, however, sex differences disappear when symptom scores are adjusted for known sex differences in controls [total score, $F(2,230)=0.77, p=0.46$, Cohen's $d=0.01$ or domain scores $[F(4,227)=1.52, p=0.197$; Cohen's $d=0.07-0.18]$.

Conclusion: Although those with prior concussions report more symptoms (but do not differ on neurocognition), this study does not support sex differences with cognition or symptoms in adolescent athletes with prior concussions.
\end{abstract}

Abstract Word Count: 200

Key words: concussion; mild traumatic brain injury; cumulative; lingering; pediatric; gender 


\section{Introduction}

Adolescents sustain concussions at higher rates compared to most other age groups, ${ }^{1,2}$ with concussion accounting for $10 \%$ of all injuries in high school athletics ${ }^{3}$ and estimates suggesting that the number of identified concussions in high school athletics may have increased over time. ${ }^{4}$ Although concussions in sports are most often associated with a temporary neurophysiological disruption of cellular signalling, ${ }^{5}$ there is concern that sustaining numerous concussions will result in long-term sequelae, often referred to as lingering or cumulative effects. In the existing literature, there are mixed reports of potential cumulative effects from previous concussions on neurocognitive functioning that range from no detected effect ${ }^{6-10}$ to measureable effects ${ }^{11-13}$. A recent study with a large sample of adolescent elite hockey players demonstrated that those with multiple prior concussions did not differ in neurocognitive function compared to those with no prior concussion. ${ }^{14}$ However, players with two or more prior concussions did report more symptoms at baseline testing compared to those with zero or one prior concussion. ${ }^{14}$ This study did not, however, examine whether there were differences between males and females with a prior concussion (i.e., sex or gender*1 differences). ${ }^{14}$

There is some evidence that female athletes are at a higher risk of concussion than males in many sports. ${ }^{4,15}$ There does not appear to be sex differences with total symptom reporting following a concussion in adolescents, ${ }^{16-18}$ however, the types of symptoms endorsed may differ between males and females..$^{18}$ In samples that contain both adolescents and adults, there are several studies suggesting that females report higher levels of symptoms ${ }^{19,20}$ or have more neurocognitive problems ${ }^{21-24}$ than males in the acute and sub-acute periods following a concussion. In adult-only samples, sex differences beyond the acute/sub-acute period following a concussion are not generally supported ${ }^{25,26}$ but there is a suggestion that multiple prior concussions may result in worse visual memory in females compared to males. ${ }^{22}$

There is limited literature available on whether prior concussions result in differential effects on male and female adolescents. The purpose of this study was to examine whether there are sex differences with neurocognitive functioning and symptom reporting in adolescent athletes with or without a history of a concussion. It was hypothesized that females with a prior concussion would have worse cognition and more symptoms than males with a history of a concussion.

\section{Participants and Study Design}

\section{Methods}

Potential participants ( $N=768$ ) were recruited for baseline testing (pre-season) as part of a 44team cohort study on concussion outcome. ${ }^{27-29}$ This sample has been previously reported but combined the males and females. ${ }^{14}$ Inclusion criteria for baseline testing were: cleared to play at the beginning of the 2011-2012 hockey season; written informed assent/consent to participate (player assent and consent from one parent or guardian); players participating in the Bantam (ages 13-14) or Midget (15-17 years) leagues only; players in the most elite $20 \%$ of divisions of play (AA, AAA); agreement of the player's head coach to participate in the study; and agreement of the team therapist to collect information about individual player participation and injury throughout the season as part of the larger cohort study.

Players were excluded from this study if they did not have baseline testing $(n=25)$, did not complete the background questionnaire with injury history $(n=28)$, identified themselves as having English as a second language $(n=11)$, endorsed having attention or learning problems $(n=26)$, endorsed having a concussion within six months before the baseline testing $(n=29)$ (the latter three items were

\footnotetext{
*1 The use of terms "sex" versus "gender" remains debatable. "Gender" is an appropriate term for this study because differences on neurocognitive testing and/or symptom reporting are likely biopsychosocial, not purely biological. However, we asked participants to identify their "sex" (male or female) when filling out the demographic information. As a result, we will use the term "sex" in this paper to refer to males and females.
} 
collected on ImPACT), or had suspected invalid test profiles flagged by the ImPACT program (test type identified as "baseline++"; $n=34$ ). The removal of those factors, such as English as a second language, inattention, and learning disorders, was done in order to remove extraneous factors that could negatively impact test performance ${ }^{30}$. As well, excluding those with an injury within the past six months was done to minimize the chances of differences being attributed to a recent injury (rather than longerterm effects). The University Conjoint Health Research Ethics Board granted approval for this study (Ethics ID E-24026).

Instruments

Reporting of previous concussions was completed using a pre-season questionnaire (PSQ), which is a previously validated measure used in injury surveillance studies in youth ice hockey by this research group. ${ }^{14,31-33}$ The PSQ is a self-report paper-and-pencil questionnaire completed with the assistance of parents/guardians. Families were sent the PSQ approximately 2-3 weeks before the baseline assessments, with athletes bringing the completed questionnaire to the pre-season testing session. The PSQ designed to collect participant demographics, current sport participation, previous medical history, and previous concussion history (i.e., asked 'Have you ever had a concussion or been 'knocked out' or had your 'bell rung'?").

Baseline assessment for all players prior to starting the hockey season included the Immediate Post-Concussion Assessment and Cognitive Testing (ImPACT) battery. ImPACT is a brief computerized screen of cognitive abilities (i.e., not a neuropsychological assessment) that has been used previously in several studies investigating neurocognitive functioning following concussive injuries. ${ }^{7,13,14,34-39}$ ImPACT includes six tests/modules (i.e., word discrimination, design memory, $x^{\prime}$ s and o's, symbol match, color match, three letters) that yield five composite/domain scores (i.e., verbal memory, visual memory, visual-motor, reaction time, and impulse control) with demographically-adjusted (i.e., age and sex) percentile scores based on the ImPACT normative data. ImPACT also includes a subjectively-rated postconcussion scale (PCS) that contains 22 symptoms being rated from 0 (none) to 6 (severe). Participants complete the PCS during the baseline (pre-season) assessment, with symptom ratings being provided on the computer before completing the neurocognitive testing. For this study, both total symptom scores and four symptom domain scores [Cognitive-Sensory, Sleep-Arousal, VestibularSomatic, and Affective (based upon factor analysis from Kontos et $\mathrm{al}^{40}{ }^{4}$ )] were included. Unlike the composite scores, the PCS does not provide normative-based comparisons (only raw scores). Analysis

Prior to analyses, ImPACT percentile scores were uniformly transformed into T scores (mean=50, $S D=10$ ) to facilitate the use of parametric statistical methods. To account for known sex differences in symptom ratings even in the absence of an injury ${ }^{40}$ (and in the anticipation that males and females without a prior concussion would differ in symptom reporting), standardized Z scores were computed for those with a prior concussion using the symptom ratings from the same-sex group without a prior concussion [i.e., for males, $Z$ score $=($ raw score-mean)/standard deviation, where mean and standard deviation are derived from males with zero prior concussions ( $n=309)$; for females, $Z$ score=(raw scoremean)/standard deviation, where mean and standard deviation are derived from females with zero prior concussions $(n=73)]$.

Student's $t$ tests were used to compare demographic variables between males and females. Gender x Prior Concussions Groups (2x2) multivariate analyses of variance (MANOVA) were used for comparisons of the five ImPACT composite scores, with planned follow-up ANOVAs for any significant group effects. Comparisons for total symptom scores were completed using a Gender $x$ Prior Concussions Groups ( $2 \times 2$ ) analyses of covariance (ANCOVA), with age as the covariate. Comparisons for domain symptom scores were completed using a Gender x Prior Concussions Groups (2x2) multivariate analyses of covariance (MANCOVA), with age as the covariate. 
Males and females with prior concussion - 6

Analyses were completed using IBM SPSS Statistics 19.0. Significance for analyses was set $a$ priori at $p<.05$. Effect sizes were computed using Cohen's $d$ (interpretation of effect sizes: $d<.20=$ very small; $\mathrm{d}=.20-.49=$ small; $\mathrm{d}=.50-.79=$ medium; $\mathrm{d} \geq .80=$ (arge).

\section{Results}

Participants $(n=615)$ ranged in age from 13.0-17.9 years old (mean age $=15.5,95 \% \mathrm{Cl}=15.4-15.6)$. Male hockey players ( $n=517,84 \%$ of sample) had a mean age of 15.5 years $(95 \% \mathrm{Cl}=15.4-15.6)$, with $84.7 \%$ right handed, and $69.4 \%$ in the Midget age group (ages 15-17). At least one prior concussion was reported in $40.2 \%(n=208)$ of the males. The median time since last concussion in the males was 22.0 months (range $=6-177)$. Female hockey players ( $n=98,16 \%$ of sample) had a mean age of 15.3 years (95\% Cl=15.0-15.6), with 91.8\% right handed, and 55.1\% in the Midget age group. At least one prior concussion was reported in $25.5 \%(n=25)$ of the females. The median time since last concussion in the females was 16.5 months (range=7-58).

Although significantly more males $(40.2 \%)$ than females $(25.5 \%)$ reported a prior concussion $\left[\chi^{2}(1)=7.56, p=0.006\right]$, there was not a significant difference in the distribution of prior concussions between males ( $n=208 ; 81.7 \%$ had one, $15.4 \%$ had two, $2.9 \%$ had three) and females [ $n=25 ; 80.0 \%$ had one, $16.0 \%$ had two, $4.0 \%$ had three; Mann-Whitney $U=2551, p=.889]$. The median time since last concussion in the females did not differ significantly compared to the males [ $t(137)=1.47, p=0.143]$. Those athletes with a prior concussion were significantly older than those without a concussion for both the males $[t(515)=2.25, p=.025]$ and females $[t(96)=2.54, p=.013]$, confirming the importance of using either demographically-adjusted scores (cognitive domains) or using age as a covariate (symptom ratings).

ImPACT Composite Scores

Performance on the five ImPACT composite scores, stratified by males and females, are presented in Table 1. In those without a prior concussion, there was not a significant difference between males and females on ImPACT performance $[F(5,376)=1.33, p=0.252$, partial eta squared $=0.017]$. When comparing males and females with a history of concussion, there was not a significant sex difference on ImPACT composite scores $[F(5,227)=1.40, p=0.227$, partial eta squared $=0.030]$. In the males only, there was not a significant difference with ImPACT composite scores when comparing those without versus those with a prior concussion $[F(5,511)=0.71, p=0.615$, partial eta squared $=0.007]$. In the females only, there was not a significant difference with performance on ImPACT in those without compared to those with a prior concussion $[F(5,92)=0.52, p=0.758$, partial eta squared $=0.028]$.

\section{Symptom Ratings - Raw Scores}

Symptom ratings for males and females, including total and domain scores, are presented in Table 2. In players without a history of concussion, fewer total symptoms were endorsed in males (mean=4.4, $95 \% \mathrm{Cl}=3.7-5.1)$ than in females [mean=7.0, 95\% $\mathrm{Cl}=4.9-9.1 ; \mathrm{F}(2,379)=4.13, \mathrm{p}=0.017$, partial eta squared=0.021; Cohen's $d=0.37$ ]. MANCOVA suggests that there were significant differences between males and females on the four PCS domains $[F(4,376)=2.67, p=0.032]$. Follow-up analyses indicate that males without a history of concussion endorsed fewer Cognitive-Sensory $[F(1,379)=8.81, p=0.003$; Cohen's d=0.38], Sleep-Arousal $[F(1,379)=8.21, p=0.004$; Cohen's $d=0.37]$, and Affective $[F(1,379)=4.54$, $p=0.034$; Cohen's $d=0.28$ ] symptoms than females without a history of concussion [Vestibular-Somatic, $F[1,379]=1.04, p=0.308$; Cohen's $d=0.13]$. In those with a history of concussion, there was not a statistical difference between males (mean $=6.3,95 \% \mathrm{Cl}=5.2-7.4$ ) and females (mean=10.1, 95\% $\mathrm{Cl}=5.3$ 14.9 ) on total symptom ratings $[F(2,230)=3.01, p=0.051$, partial eta squared $=0.026]$, but there was a small effect size (Cohen's $d=0.42$ ) suggesting that power may have been limited to detect statistical differences. MANCOVA suggests that there were not statistically significant differences between males and females on symptom ratings across the four domains $[F(4,227)=1.52, p=0.197]$, however, effect sizes range from small (Cohen's $d=0.30$, Vestibular-Somatic) to medium (Cohen's $d=.50$, Affective) and suggest higher domain ratings in females. 
Table 1. Neurocognitive performance in male and female adolescent athletes with or without history of prior concussions.

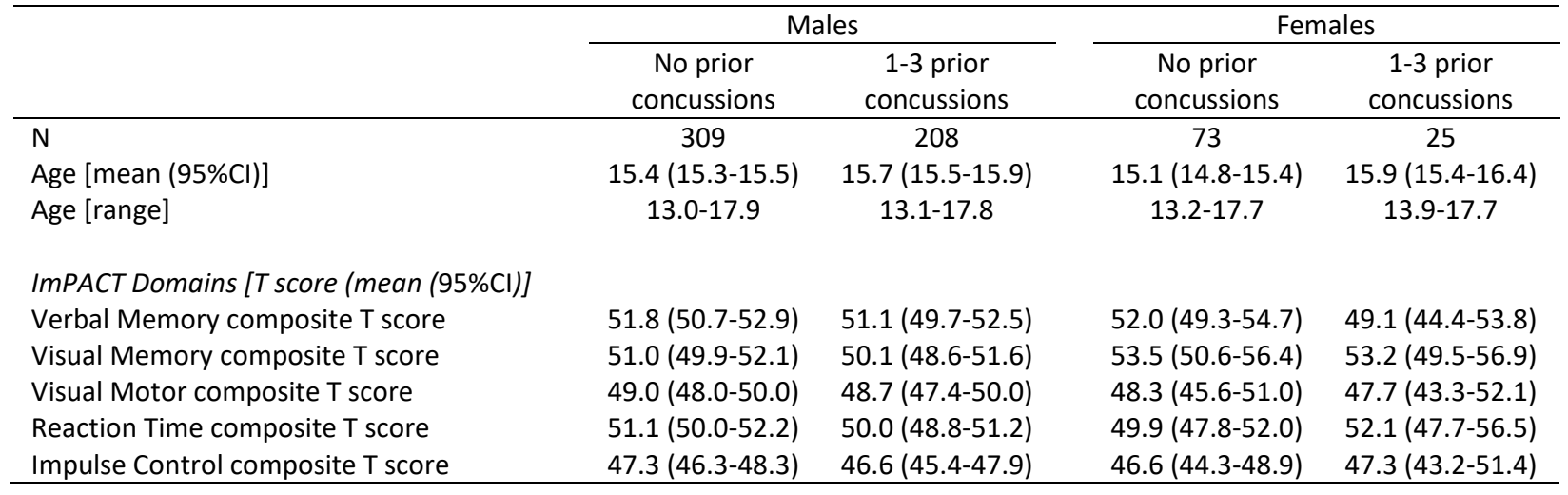

Table note: $95 \% \mathrm{Cl}=95 \%$ confidence interval. ImPACT=Immediate Postconcussion Assessment and Cognitive Testing. PCS=postconcussion scale. Values presented for ImPACT domain scores are age- and sex-adjusted T scores (mean=50, standard deviation=10). For ImPACT composite scores, higher scores reflect better performance relative to age and sex norms.

Table 2. Symptom reporting in male and female adolescent athletes with or without history of prior concussions.

\begin{tabular}{|c|c|c|c|c|}
\hline & \multicolumn{2}{|c|}{ Males } & \multicolumn{2}{|c|}{ Females } \\
\hline & $\begin{array}{c}\text { No prior } \\
\text { concussions }\end{array}$ & $\begin{array}{c}\text { 1-3 prior } \\
\text { concussions }\end{array}$ & $\begin{array}{c}\text { No prior } \\
\text { concussions }\end{array}$ & $\begin{array}{c}\text { 1-3 prior } \\
\text { concussions }\end{array}$ \\
\hline $\mathrm{N}$ & 309 & 208 & 73 & 25 \\
\hline Age [mean $(95 \% \mathrm{Cl})]$ & $15.4(15.3-15.5)$ & $15.7(15.5-15.9)$ & $15.1(14.8-15.4)$ & $15.9(15.4-16.4)$ \\
\hline Age [range] & $13.0-17.9$ & $13.1-17.8$ & $13.2-17.7$ & $13.9-17.7$ \\
\hline \multicolumn{5}{|c|}{ Symptom Ratings [raw score (mean $(95 \% \mathrm{Cl})$ ] } \\
\hline Total Symptom Score & $4.4(3.7-5.1)$ & $6.3(5.2-7.4)$ & $7.0(4.9-9.1)$ & $10.1(5.3-14.9)$ \\
\hline Cognitive-Sensory domain & $1.1(0.9-1.3)$ & $1.8(1.4-2.2)$ & $2.0(1.3-2.7)$ & $2.7(1.3-4.1)$ \\
\hline Sleep-Arousal domain & $1.7(1.4-2.0)$ & $2.4(2.0-2.8)$ & $2.6(1.8-3.4)$ & $3.5(2.0-5.0)$ \\
\hline Vestibular-Somatic domain & $0.6(0.4-0.8)$ & $0.9(0.6-1.2)$ & $0.8(0.3-1.3)$ & $1.6(0.5-2.7)$ \\
\hline Affective domain & $0.8(0.6-1.0)$ & $1.0(0.7-1.3)$ & $1.3(0.7-1.9)$ & $2.2(0.3-4.1)$ \\
\hline \multicolumn{5}{|l|}{ Symptom Ratings [Z score (mean $(95 \% \mathrm{Cl}])$} \\
\hline Total Symptom Z Score & -- & $0.31(0.13-0.49)$ & -- & $0.38(-0.16-0.92)$ \\
\hline Cognitive-Sensory domain Z score & -- & $0.32(0.13-0.51)$ & -- & $0.23(-0.35-0.81)$ \\
\hline Sleep-Arousal domain Z score & -- & $0.32(0.14-0.50)$ & -- & $0.21(-0.33-0.75)$ \\
\hline Vestibular-Somatic domain Z score & -- & $0.22(0.03-0.41)$ & -- & $0.36(-0.22-0.94)$ \\
\hline Affective domain Z score & -- & $0.12(-0.03-0.27)$ & -- & $0.31(-0.15-0.77)$ \\
\hline
\end{tabular}

Table note: $95 \% \mathrm{Cl}=95 \%$ confidence interval. ImPACT=Immediate Postconcussion Assessment and Cognitive Testing. PCS=postconcussion scale. "Total symptom score" is based on summing all of the ratings of severity for each item (0-6), where higher values represent higher ratings of symptom severity. Raw symptom ratings presented are statistically adjusted for age differences across the groups. Domains of symptom scores are based on factor structure of the post-concussion scale (Kontos et al., $\left.{ }^{38}\right)$. Z-score computations of symptom scores are based on using the means and standard deviations from those same-sex adolescents with zero prior concussions, with mean scores then statistically adjusted for age differences across the groups. $Z$ scores have a mean $=0$ with a standard deviation ranging from -1 to +1 , with higher scores reflecting more symptoms than the 'control' (zero prior concussions) sample. 
Males and females with prior concussion - 8

In males only, those with a history of concussion had significantly higher total symptoms than those without a prior concussion $[F(2,514)=5.60, p=0.004$, partial eta squared $=0.021$ Cohen's $d=0.26]$. MANCOVA suggests that there were significant differences on the PCS domains in those without and those with a prior concussion $[F(4,511)=2.69, p=0.031]$. Follow-up analyses indicate that males with a history of concussion endorsed higher levels of Cognitive-Sensory $[F(1,514)=8.56, p=0.004$; Cohen's $d=0.27]$, Sleep-Arousal $[F(1,514)=7.73, p=0.006$; Cohen's $d=0.26]$, and Vestibular-Somatic $[F(1$, $514)=4.98, p=0.026$; Cohen's $d=0.18$ ] symptoms than males without a history of concussion [Affective, $F[1,514]=1.70, p=0.193 ;$ Cohen's $d=0.11]$.

In females only, those with and those without a prior concussion did not have significantly different total symptom scores $[F(2,95)=0.97, p=0.383$, partial eta squared $=0.020]$, although a small effect size was present (Cohen's $d=0.41$ ) suggesting that power may have been limited to detect statistical differences. MANCOVA suggested that there were not statistically significant differences on the four PCS domains in those with a prior concussion $[F(4,92)=0.80, p=0.53]$; however, small effect sizes ranging from Cohen's $d=0.29$ (Cognitive-Sensory, Sleep-Arousal) to Cohen's $d=0.42$ (Affective) suggest higher domain ratings in those with a prior concussion (and possibly a lack of power to detect statistical differences).

Symptom Ratings - Standardized Scores

When examining standardized $Z$ scores for the symptom ratings in those with a prior concussion, based on using the performance of the same-sex sample with zero concussions, there was not a statistically significant difference between males and females for total symptoms $[F(2,230)=0.77$, $p=0.463$, partial eta squared $=0.007$; Cohen's $d=0.01]$. When examining standardized $Z$ scores for the four symptom domains, again there were not statistical differences between males and females with history of concussion $[F(4,227)=1.52, p=0.197$; Cohen's $d=0.07-0.18]$.

\section{Discussion}

The purpose of this study was to examine whether there are sex differences in adolescent athletes with a history of prior concussive injuries. As researchers and clinicians strive to consider whether there are developmental differences from concussions, it is also important to consider whether there are sex differences from prior concussions as well. In this sample of elite 13-17-year-old hockey players with baseline testing at the start of the season, the results suggest that there were no differences in cognition or symptoms between females and males with a history of prior concussions (when accounting for known sex differences in healthy controls).

To date, there has been a paucity of studies on differential sex effects from prior concussions on neurocognitive functioning. In this sample, males and females with a prior history of concussion did not demonstrate sex differences on the rapid screen of neurocognitive abilities (i.e., verbal memory, visual memory, visual motor, reaction time, and impulse control composites from ImPACT). The absence of sex differences on ImPACT performance builds upon the previous findings in this sample that there are no neurocognitive differences in those adolescent athletes with zero, one, or two or more prior concussions. ${ }^{14}$ To date, there has not been research examining whether adolescents with a history of concussions demonstrate sex differences on neurocognitive testing. In samples containing both adolescent and adults, there is some literature that suggests females have more neurocognitive problems in the acute and subacute periods compared to males, ${ }^{20,21,23,24}$ but explorations of longerterm differences or 'cumulative effects' are lacking.

Research on NCAA athletes with prior concussions suggests the potential for sex differences in neurocognitive abilities in those with injuries more than 3 years before testing. ${ }^{22}$ When considering athletes who were more than three years post-injury, females performed statistically better than males on all ImPACT composite scores (verbal memory, visual memory, processing speed, and reaction time). However, it is also important to note that the Covassin et al. ${ }^{22}$ study examined raw scores instead of demographically-adjusted values, with the same group previously demonstrating that raw scores 
contain sex differences (i.e., males outperform females on visual memory tasks, females outperform males on verbal memory tasks ${ }^{41}$ ). The presence of sex differences on ImPACT performance is known and the need to use demographically-adjusted scores is evident based on the standardization data put forth by the test publisher (i.e., normative values for composite scores are stratified by age and sex), therefore, it is uncertain whether normal sex differences found on ImPACT are also driving (or partially driving) sex differences in those with remote concussions.

The examination of sex differences on symptom reporting in adolescent athletes with prior concussions provides several important findings. First, there are sex differences in symptom reporting in those without prior injuries. That is, healthy females adolescent athletes report higher levels of cognitive-sensory, sleep-arousal, and affective symptoms (as well as total symptoms) at baseline (preseason) compared to males (there was not a difference between males and females with reporting of vestibular-somatic symptoms). Interestingly, these results are largely consistent with several studies showing sex differences in symptom reporting, ${ }^{18,40}$ but do differ somewhat from other studies that suggest no sex differences in a small sample of healthy adolescents ${ }^{16}$ or healthy adults. ${ }^{26,42}$ Second, both males and females with a history of prior concussions report more baseline (pre-season) symptoms than their same-sex counterparts without prior concussions. Third, and perhaps most important for the purpose of this study, the difference in symptom reporting between those with and without a history of prior concussions does not differ between males and females when using sex-adjusted scores in the analyses. When using the athletes with zero concussions as a control group and standardizing symptom ratings separately for males and females, sex differences in those with a history of prior concussions are washed out. To our knowledge, no other study has explored sex differences using sex-adjusted standardized symptom reporting, although the methodology obviously provides important results.

There are some limitations that should be addressed with this study. First, and perhaps most important, this is a cross-sectional design that cannot support a causal relationship between past injuries and current functioning. It is impossible to know whether the remote concussive injuries led to the higher levels of symptom reporting. Without evidence of direct causation, attribution of elevated symptoms to remote injuries is far from a fait accompli. Second, this is a homogeneous sample of elite hockey players that may not be representative of all adolescents (e.g., those with attention or learning problems or those who spoke English as a second language were removed from the analyses). This was done purposely to answer a specific question about sex differences and prior concussion, knowing that other factors can negatively impact test performance (e.g., Elbin et al., ${ }^{30}$ ) and would affect the specific research question. Because this study excluded those who were within six months of an injury (done to remove the effects of ongoing recovery), this sample is also not representative of those athletes who are acutely or sub-acutely injured. As well, this sample may not be representative of athletes in other sports. Third, the ImPACT battery is considered a rapid screen of neurocognitive functioning and is not a neuropsychological assessment. It is possible that more in-depth testing covering a wider range of cognitive domains could reveal important neurocognitive effects not found using this computerized screen. Fourth, this was a small sample of females, particularly in comparison to the sample of males. As a result, analyses were presented for those with a range of prior concussions (between one and three) and more fine-grained analyses with the females would not be possible (e.g., single prior concussion versus multiple prior concussions). As well, there were several statistical analyses with non-significant differences, suggesting the small sample size of females with prior concussions may have been under powered. Of course, the effect sizes for most comparisons were small (except a medium effect size for affective symptoms between males and females with a prior concussion), so having more power to detect statistical differences may not result in clinically meaningful findings.

Overall, the results of this study do not support a differential sex effect in adolescent athletes who have a prior history of concussion. Males and females with a prior history of concussion do not differ with neurocognitive functioning. When accounting for known sex differences in symptom reporting 
(females report more symptoms than males), there were no sex differences in those with a prior history of concussion. Of course, this research is only presenting differences in those with a history of injury, so the results cannot support a direct causation between a past injury and current presentation. Further research into the long-term effects of concussions in pediatric samples is needed, particularly with study designs that can better elucidate whether there are truly causal effects of injuries on future presentation (not just an association).

\section{References}

1. Cassidy JD, Carroll L, Peloso PM, et al. Incidence, risk factors and prevention of mild traumatic brain injury: results of the WHO Collaborating Centre Task Force on Mild Traumatic Brain Injury. Journal of Rehabilitation Medicine. Feb 2004(43 Suppl):28-60.

2. NIH Consensus Development Panel. Consensus conference. Rehabilitation of persons with traumatic brain injury. NIH Consensus Development Panel on Rehabilitation of Persons With Traumatic Brain Injury. JAMA. Sep 8 1999;282(10):974-983.

3. Gessel LM, Fields SK, Collins CL, Dick RW, Comstock RD. Concussions among United States high school and collegiate athletes. J Athl Train. Oct-Dec 2007;42(4):495-503.

4. Lincoln AE, Caswell SV, Almquist JL, Dunn RE, Norris JB, Hinton RY. Trends in concussion incidence in high school sports: a prospective 11-year study. Am J Sports Med. May 2011;39(5):958-963.

5. Giza CC, Hovda DA. The neurometabolic cascade of concussion. Journal of Athletic Training. Sep 2001;36(3):228-235.

6. Bijur PE, Haslum M, Golding J. Cognitive outcomes of multiple mild head injuries in children. J Dev Behav Pediatr. Jun 1996;17(3):143-148.

7. Iverson GL, Brooks BL, Lovell MR, Collins MW. No cumulative effects for one or two previous concussions. Br J Sports Med. Jan 2006;40(1):72-75.

8. Theriault M, De Beaumont L, Tremblay S, Lassonde M, Jolicoeur P. Cumulative effects of concussions in athletes revealed by electrophysiological abnormalities on visual working memory. $J$ Clin Exp Neuropsychol. Jan 2011;33(1):30-41.

9. Thornton $A E$, Cox DN, Whitfield K, Fouladi RT. Cumulative concussion exposure in rugby players: neurocognitive and symptomatic outcomes. J Clin Exp Neuropsychol. May 2008;30(4):398-409.

10. Bruce JM, Echemendia RJ. History of multiple self-reported concussions is not associated with reduced cognitive abilities. Neurosurgery. Jan 2009;64(1):100-106; discussion 106.

11. Elbin RJ, Covassin T, Hakun J, et al. Do brain activation changes persist in athletes with a history of multiple concussions who are asymptomatic? Brain Inj. 2012;26(10):1217-1225.

12. Moser RS, Schatz P, Jordan BD. Prolonged effects of concussion in high school athletes. Neurosurgery. Aug 2005;57(2):300-306; discussion 300-306.

13. Iverson GL, Echemendia RJ, Lamarre AK, Brooks BL, Gaetz MB. Possible lingering effects of multiple past concussions. Rehabil Res Pract. 2012;2012:316575.

14. Brooks BL, McKay C, Mrazik M, Barlow KM, Meeuwisse W, Emery CA. Subjective, but not objective, lingering effects of multiple past concussions in adolescents. $J$ Neurotrauma. in press.

15. Dick RW. Is there a gender difference in concussion incidence and outcomes? Br J Sports Med. May 2009;43 Suppl 1:i46-50.

16. Zuckerman SL, Solomon GS, Forbes JA, Haase RF, Sills AK, Lovell MR. Response to acute concussive injury in soccer players: is gender a modifying factor? J Neurosurg Pediatr. Dec 2012;10(6):504-510.

17. Preiss-Farzanegan SJ, Chapman B, Wong TM, Wu J, Bazarian JJ. The relationship between gender and postconcussion symptoms after sport-related mild traumatic brain injury. $P M R$. Mar 2009;1(3):245-253.

18. Frommer $L$, Gurka KK, Cross KM, Ingersoll CD, Comstock RD, Saliba SA. Sex differences in concussion symptoms of high school athletes. J Athl Train. Jan-Feb 2011;46(1):76-84. 
19. Kontos AP, Dolese A, Elbin RJ, Covassin T, Warren BL. Relationship of soccer heading to computerized neurocognitive performance and symptoms among female and male youth soccer players. Brain Inj. 2011;25(12):1234-1241.

20. Dougan BK, Horswill MS, Geffen GM. Athletes' Age, Sex, and Years of Education Moderate the Acute Neuropsychological Impact of Sports-Related Concussion: A Meta-analysis. J Int Neuropsychol Soc. Feb 4 2013:1-17.

21. Covassin T, Elbin RJ, Harris W, Parker T, Kontos A. The role of age and sex in symptoms, neurocognitive performance, and postural stability in athletes after concussion. Am J Sports Med. Jun 2012;40(6):1303-1312.

22. Covassin $\mathrm{T}$, Elbin R, Kontos A, Larson E. Investigating baseline neurocognitive performance between male and female athletes with a history of multiple concussion. J Neurol Neurosurg Psychiatry. Jun 2010;81(6):597-601.

23. Colvin AC, Mullen J, Lovell MR, West RV, Collins MW, Groh M. The role of concussion history and gender in recovery from soccer-related concussion. Am J Sports Med. Sep 2009;37(9):1699-1704.

24. Broshek DK, Kaushik T, Freeman JR, Erlanger D, Webbe F, Barth JT. Sex differences in outcome following sports-related concussion. J Neurosurg. May 2005;102(5):856-863.

25. Tsushima WT, Lum M, Geling O. Sex differences in the long-term neuropsychological outcome of mild traumatic brain injury. Brain Inj. Sep 2009;23(10):809-814.

26. Covassin T, Schatz $P$, Swanik CB. Sex differences in neuropsychological function and post-concussion symptoms of concussed collegiate athletes. Neurosurgery. Aug 2007;61(2):345-350; discussion 350341.

27. McKay C, Brooks B, Mrazik M, Jubinville A, Meeuwisse M, Emery C. Baseline Immediate PostConcussion Assessment and Cognitive Testing Values and test-retest reliability in elite Canadian youth ice hockey players [Abstract]. Clin J Sport Med. 2012;22(3):307.

28. Blake TA, Taylor KA, Woollings KY, et al. Sport Concussion Assessment Tool, Version 2, normative values and test-retest reliability in elite youth ice hockey [Abstract]. Clin J Sport Med.

2012;22(3):307.

29. Schneider KJ, Emery CA, Kang J, Blake TA, Meeuwisse WH. Are Sport Concussion Assessment Tool, Version 2, scores different for youth ice hockey players with and without a previous history of concussion? [Abstract]. Clin J Sport Med. 2012;22(3):307.

30. Elbin RJ, Kontos AP, Kegel N, Johnson E, Burkhart S, Schatz P. Individual and combined effects of LD and ADHD on computerized neurocognitive concussion test performance: Evidence for separate norms. Arch Clin Neuropsychol. in press.

31. Emery CA, Meeuwisse WH. Injury rates, risk factors, and mechanisms of injury in minor hockey. Am J Sports Med. Dec 2006;34(12):1960-1969.

32. Emery CA, Kang J, Shrier I, et al. Risk of injury associated with body checking among youth ice hockey players. JAMA. Jun 9 2010;303(22):2265-2272.

33. Emery C, Kang J, Shrier I, et al. Risk of injury associated with bodychecking experience among youth hockey players. CMAJ. Aug 9 2011;183(11):1249-1256.

34. Covassin T, Elbin RJ, 3rd, Larson E, Kontos AP. Sex and age differences in depression and baseline sport-related concussion neurocognitive performance and symptoms. Clin J Sport Med. Mar 2012;22(2):98-104.

35. Thomas DG, Collins MW, Saladino RA, Frank V, Raab J, Zuckerbraun NS. Identifying neurocognitive deficits in adolescents following concussion. Acad Emerg Med. Mar 2011;18(3):246-254.

36. Iverson GL, Brooks BL, Collins MW, Lovell MR. Tracking neuropsychological recovery following concussion in sport. Brain Inj. Mar 2006;20(3):245-252.

37. Schatz P. Long-term test-retest reliability of baseline cognitive assessments using ImPACT. Am J Sports Med. Jan 2010;38(1):47-53. 
38. Iverson GL, Lovell MR, Collins MW. Interpreting change on ImPACT following sport concussion. Clin Neuropsychol. Nov 2003;17(4):460-467.

39. Maroon JC, Lovell MR, Norwig J, Podell K, Powell JW, Hartl R. Cerebral concussion in athletes: evaluation and neuropsychological testing. Neurosurgery. Sep 2000;47(3):659-669; discussion 669672.

40. Kontos AP, Elbin RJ, Schatz $\mathrm{P}$, et al. A revised factor structure for the post-concussion symptom scale: baseline and postconcussion factors. Am J Sports Med. Oct 2012;40(10):2375-2384.

41. Covassin T, Swanik CB, Sachs M, et al. Sex differences in baseline neuropsychological function and concussion symptoms of collegiate athletes. Br J Sports Med. Nov 2006;40(11):923-927; discussion 927.

42. Piland SG, Ferrara MS, Macciocchi SN, Broglio SP, Gould TE. Investigation of baseline self-report concussion symptom scores. J Athl Train. May-Jun 2010;45(3):273-278. 